\title{
Profiling the circulating miRNAs in mice exposed to gram-positive and gram-negative bacteria by Illumina small RNA deep sequencing
}

Cheng-Shyuan Rau', Shao-Chun Wư ${ }^{2}$, Johnson Chia-Shen Yang ${ }^{3}$, Tsu-Hsiang Lư ${ }^{3}$ Yi-Chan Wu ${ }^{3}$, Yi-Chun Chen ${ }^{3}$, Siou-Ling Tzeng ${ }^{3}$, Chia-Jung $\mathrm{Wu}^{3}$ and Ching-Hua Hsieh ${ }^{3^{*}}$

\begin{abstract}
Background: We profiled the expression of circulating microRNAs (miRNAs) in mice using Illumina small RNA deep sequencing in order to identify the miRNAs that may potentially be used as biomarkers to distinguish between gram-negative and gram-positive bacterial infections.

Results: Recombinant-specific gram-negative pathogen Escherichia coli (Xen14) and gram-positive pathogen Staphylococcus aureus (Xen29) were used to induce bacterial infection in mice at a concentration of $1 \times 10^{8}$ bacteria/100 $\mu \mathrm{L}$ of phosphate buffered saline (PBS). Small RNA libraries generated from the serum of mice after exposure to PBS, Xen14, Xen29, and Xen14 + Xen29 via the routes of subcutaneous injection (I), cut wound (C), or under grafted skin (S) were analyzed using an Illumina HiSeq2000 Sequencer. Following exposure to gram-negative bacteria alone, no differentially expressed miRNA was found in the injection, cut, or skin graft models. Exposure to mixed bacteria induced a similar expression pattern of the circulating miRNAs to that induced by gram-positive bacterial infection. Upon gram-positive bacterial infection, 9 miRNAs (mir-193b-3p, mir-133a-1-3p, mir-133a-2-3p, mir-133a-1-5p, mir-133b-3p, mir-434-3p, mir-127-3p, mir-676-3p, mir-215-5p) showed upregulation greater than 4 -fold with a $p$-value $<0.01$. Among them, mir-193b-3p, mir-133a-1-3p, and mir-133a-2-3p presented the most common miRNA targets expressed in the mice exposed to gram-positive bacterial infection.
\end{abstract}

Conclusions: This study identified mir-193b-3p, mir-133a-1-3p, and mir-133a-2-3p as potential circulating miRNAs for gram-positive bacterial infections.

Keywords: microRNAs (miRNAs), Circulating microRNAs, Gram-positive bacteria, Gram-negative bacteria, Small RNA deep sequencing

\section{Background}

MicroRNAs (miRNAs) are small regulatory RNA molecules that are approximately 22 nucleotides long, modulate the activity of specific mRNA targets, and play important roles in a wide range of physiologic and pathologic processes [1]. Differential expression of miRNAs may help distinguish between disease states [2-4]. miRNAs are themselves active moieties and should therefore reflect physiological alterations directly, which makes them ideal biomarkers for distinguishing between diseased and healthy subjects [5].

\footnotetext{
*Correspondence: m93chinghua@gmail.com

${ }^{3}$ Department of Plastic and Reconstructive Surgery, Kaohsiung Chang Gung Memorial Hospital and Chang Gung University College of Medicine, No.123, Ta-Pei Road, Niao-Song District, Kaohsiung City 833, Taiwan

Full list of author information is available at the end of the article
}

Moreover, circulating miRNAs in the blood are remarkably stable [6] and biochemical analyses indicate that miRNAs are resistant to ribonuclease (RNase) activity, extreme $\mathrm{pH}$ and temperature, extended storage, and large numbers of freeze-thaw cycles $[7,8]$. Circulating miRNAs, which can be easily detected by non-invasive methods, have proven to be potentially valuable biomarkers for a variety of diseases $[9,10]$.

Early diagnosis of potential bacterial infection has become the key approach to dealing with the infection illness and to timely correction of the associated complications. Microbiological culture is the gold standard in distinguishing sepsis from other non-infectious diseases, but this technique is always time-consuming and can delay treatment. 
Circulating miRNAs have been reported to be associated with sepsis $[11,12]$ and various infection diseases, such as viral exposure [13,14], tuberculosis [15], and parasites $[16,17]$. A distinct circulating miRNA expression was also found in mice exposed to lipopolysaccharide (LPS) originating in the wall of gram-negative bacteria, as well as to lipoteichoic acid (LTA), a major component of grampositive bacterial walls $[18,19]$. In addition, increasing evidence has suggested that circulating miRNAs also have important biologic functions [20]. Recently, the Illumina deep sequencing platform has been used for efficient miRNA discovery, and it is widely used to generate small RNA profiles in various organisms. In this study, we profiled the expression of the circulating miRNAs in a mouse model of gram-negative and/or gram-positive bacterial infection using Illumina small RNA deep sequencing.

\section{Methods}

\section{Experimental design}

Male C57BL/6 mice (age, 10-12 weeks; weight, 30-35 g) were purchased from BioLasco (Yi-Lan, Taiwan). The mice were anesthetized by intraperitoneal injection of an anesthetic cocktail consisting of $0.1 \mathrm{mg} / \mathrm{g}$ ketamine and $0.01 \mathrm{mg} / \mathrm{g}$ xylazine. The anesthetized mice were restrained in a supine position on a heated pad to maintain body temperature at $37^{\circ} \mathrm{C}$. Recombinant-specific gram-negative pathogen Escherichia coli (Xen14) and gram-positive pathogen Staphylococcus aureus (Xen29) purchased from Caliper (Caliper, USA) were used to induce bacterial infection in the mice at a concentration of $1 \times 10^{8}$ bacteria/100 $\mu \mathrm{L}$ of phosphate buffered saline (PBS). To create mixed gram-negative and gram-positive bacterial infection, $1 \times 10^{8}$ Xen14 bacteria and $1 \times 10^{8}$ Xen29 bacteria/100 $\mu \mathrm{L}$ of PBS were used for wound contamination. Three animal models were used to create bacterial infection routes: subcutaneous injection (hereafter referred to as (I)), cut wound (hereafter referred to as (C)), and skin grafting (hereafter referred to as (S)). In the (I) model, E. coli and/or S. aureus suspensions were injected subcutaneously into the backs of the mice using an Fr. 25 needle. In the (C) model, a $1 \mathrm{~cm}$ incision wound was created in the midline of the back, smeared with E. coli and/or S. aureus suspension, and the wound was closed directly with a 4-0 nylon suture. In the (S) model, a $1 \times 1 \mathrm{~cm}$ rectangular full- thickness skin graft was lifted from the backs of the mice, E. coli and/or $S$. aureus suspensions were spread over the wound bed, and the skin graft was reattached and closed with a 4-0 nylon suture. An additional group of animals in each of these three models was inoculated with PBS to serve as a negative control. The mice had ad libitum access to food and water both before and after the surgery or administration of bacterial infection. The mice were killed 24. $\mathrm{h}$ after the administration of bacterial infection, and the whole blood was drawn and collected in RNAprotect Animal Blood Tubes (cat.No. 76544, Qiagen, USA) without anticoagulant. After the whole blood samples were incubated at room temperature for $15 \mathrm{~min}$, they were centrifuged at $3000 \times g$ for $10 \mathrm{~min}$, white blood cells were slowly removed from the corresponding layers, and the serum was extracted and stored at $-80^{\circ} \mathrm{C}$ before processing for RNA analyses. All the housing conditions and the surgical procedures, analgesia, and assessments were in accordance with national and institutional guidelines, and an Association for Assessment and Accreditation of Laboratory Animal Care (AAALAC)-accredited SPF facility was used. The animal protocols were approved by the Institutional Animal Care and Use Committee (IACUC) of Kaohsiung Chang Gung Memorial Hospital.

\section{RNA isolation}

Total RNA was extracted from the harvested serum using a mirVana miRNA Isolation Kit (Ambion, USA). The purified RNA yield was determined by the absorbance at $260 \mathrm{~nm}$ with an SSP-3000 NanoDrop spectrophotometer (Infinigen Biotech, USA), and RNA quality was evaluated with a BioAnalyzer 2100 system (Agilent Technologies, USA).

\section{Small RNA library preparation}

RNA smaller than 200 base pairs (bp) was enriched with the mirVana miRNA isolation kit (Ambion). The small RNA samples were sent to GeneTech Biotech Co., Ltd (GeneTech, Taiwan) for small RNA cloning. The population of miRNAs with a length of 15-30 nucleotides (nt) was passively eluted from polyacrylamide gels. The RNA was then precipitated with ethanol and dissolved in water. Small RNAs had linkers ligated to them and bar-coded cDNAs were prepared using a TruSeq Small RNA Sample Prep Kit (Illumina, USA) following the manufacturer's instructions. Briefly, $1 \mu \mathrm{g}$ of small RNA was ligated with adapters at $3^{\prime}$ and $5^{\prime}$ ends. Adapter-ligated RNA was reverse-transcribed with SuperScript II Reverse Transcriptase (Invitrogen, USA), then PCR-amplified (15 cycles). Samples were barcoded using 15 variants of the reverse primer provided with the kit. The indexing barcode of this kit after adapter ligation could significantly reduce sample bias over previous indexing/barcoding approaches where a barcode was ligated directly to the miRNA [21]. Individual libraries were analyzed on a BioAnalyzer (Agilent) for the presence of linked cDNA at the appropriate size (135$165 \mathrm{bp}$ ) and 11 bar-coded libraries were pooled into 1 sample by mixing $2.0 \mathrm{ng}$ of the 135-165 bp peak from each sample, as determined by the BioAnalyzer.

\section{Illumina small RNA deep sequencing}

Sequencing of the pooled libraries was performed in 1 lane of the Illumina HiSeq2000 Sequencer. Fifty bp single-end 
reads of the libraries were obtained. After indexing and trimming of linker sequences, those reads of at least $15 \mathrm{nt}$ in length that had less than three terminal mismatches in the sequence were sorted and counted for the following analysis. The clean reads were aligned against the reference genome indices for mouse ( $M$. musculus, UCSC $\mathrm{mm} 9$ ) provided by the Bowtie site (http://bowtie-bio. sourceforge.net/index.shtml) using BOWTIE software according to the following criteria: a $5^{\prime}$ and $3^{\prime}$ linker match of at least $15 \mathrm{nt}$ and an appropriate length (15-29 nt). The pre-miRNAs and mature miRNAs in the miRBase v.20.0 were searched with BLAST to identify Mus musculus miRNAs. To evaluate the quality of deep sequencing experiments, five miRNAs detected by small RNA deep sequencing were randomly selected and quantified by qPCR using the Applied Biosystems 7500 Real-Time PCR System (Life Technologies, USA) to confirm the upregulation of miRNA expression in Xen29 (I) group vs. Xen29 (C) group. Twenty-five femto-molar of single stranded cel-miR-39 synthesized by Invitrogen (Invitrogen) was spiked into $400 \mu \mathrm{L}$ of serum as an internal control for the expression of each miRNA.

\section{Results}

\section{Deep sequence analysis of small RNAs}

To profile the circulating miRNAs expressed during gram-negative and gram-positive bacterial infection, 12 small RNA libraries were generated from the serum of mice after exposure to PBS, Xen14, Xen29, and Xen14+ Xen29 via the routes of subcutaneous injection (I), cut wound $(\mathrm{C})$, or under grafted skin (S). These 12 small RNA libraries were indicated as Contrl (I), Xen14 (I), Xen29 (I), Xen14 + Xen29 (I), Contrl (C), Xen14 (C), Xen29 (C), Xen14 + Xen29 (C), Contrl (S), Xen14 (S), Xen29 (S), and Xen14 + Xen29 (S). The number and proportions of the categories of small RNAs found are given in the Additional file 1. In total, around 5424663 to 5999008 high-quality raw reads were obtained from the serum libraries. After filtering the low-quality sequences, empty adaptors and single-read sequences, $92.58 \%$ to $97.85 \%$ clean reads of 15-29 nt were selected for further analysis (Additional file 1: Table S1). The selected reads from these serum libraries mapped well to the mice genome, amounting to $74.19 \%$ and $79.32 \%$ of the total reads. The rest of the sequences were found to be other types of RNA, including noncoding RNA, rRNA, scRNA, snRNA, snoRNA, srpRNA, and tRNA. The size distribution of small RNAs (sRNAs) was similar in the 2 libraries, and the majority of them were from 21 to $23 \mathrm{nt}$ (Figure 1). The most abundant size class was $22 \mathrm{nt}$, which accounted for $\sim 40 \%$ of the total reads in these libraries, followed by $23 \mathrm{nt}$ for $\sim 35 \%$ (Additional file 1: Table S2). This result is typical of Dicerprocessed small RNA products and was consistent with the known $\sim 22$ nt for miRNAs.

\section{Identification of known miRNAs}

The Illumina small RNA deep sequencing approach allows us to determine the relative abundance of various miRNA families by calculating the sequencing frequency. To investigate the expression of known miRNAs during bacterial infection, identified small RNA sequences were compared with known mature miRNAs in miRBase v.20.0. The measured 814 mature miRNAs from these 12 libraries are listed (Additional file 1: Table S3). Among them, there were 52 mature miRNAs with sequence reads $\geq 400$ (Additional file 1: Table S4) and 10 mature miRNAs (mir-10b-5p, mir-133a-1-3p, mir-133a2-3p, mir-191-5p, mir-22-3p, mir-25-3p, mir-3107-5p, mir-486-5p, mir-92a-1-3p, mir-92a-2-3p) with sequence reads $\geqq 4000$ in at least 1 of the 12 twelve libraries (Additional file 1: Table S5). In these libraries, known miRNAs had a broad range of expression level; some (such as mir-486-5p and mir-3107-5p) were found to have hundreds of thousands of sequence reads, while many others had less than 20 , indicating that expression varies significantly among different miRNA families. With more than 6000 reads, 3 miRNAs (mir-486-5p, mir-3107-5p, and mir-92a-3p) were dominantly expressed in all these 12 libraries (Additional file 1: Table S6). In addition to mir-486-5p and mir-3107-5p, which have more than 200000 sequence reads in all 12 libraries, mir-92a-3p was the third most abundant miRNA with sequence reads ranging from 6659 to 16734 (Additional file 1: Table S6). Small RNA deep sequencing and qPCR results of five selected miRNAs (mir-133a-1-3p, mir-127-3p, mir-25-3p, mir-191-5p, and mir-215-5p) were generally in agreement, with a Pearson correlation value of 0.921 (Additional file 2).

\section{Differentially-expressed miRNAs after bacterial infection}

According to the changes in relative miRNA abundance between the serum libraries from the mice receiving bacterial infection (Xen14, Xen29, and Xen14 + Xen29) and PBS injection after $24 \mathrm{~h}$, the differentially-expressed miRNAs with sequence reads more than 400 in at least 1 of the libraries were selected for further comparison. It was revealed that a total of 9 miRNAs (mir-193b-3p, mir133a-1-3p, mir-133a-2-3p, mir-133a-1-5p, mir-133b-3p, mir-434-3p, mir-127-3p, mir-676-3p, mir-215-5p) showed differences greater than 4 -fold with $p$-value $<0.01$ between the 2 libraries (Table 1). Following exposure to gramnegative bacteria alone, no differentially-expressed miRNAs were found in the injection, cut, or skin graft models. Following exposure to gram-positive bacteria in the injection and skin graft models, 7 upregulated miRNAs (mir-193b-3p, mir-133a-1-3p, mir-133a-2-3p, mir-133b-3p, mir-434-3p, mir-127-3p, mir-676-3p) and 1 downregulated miRNA (mir-215-5p) were found. In addition, following exposure to gram-positive bacteria 


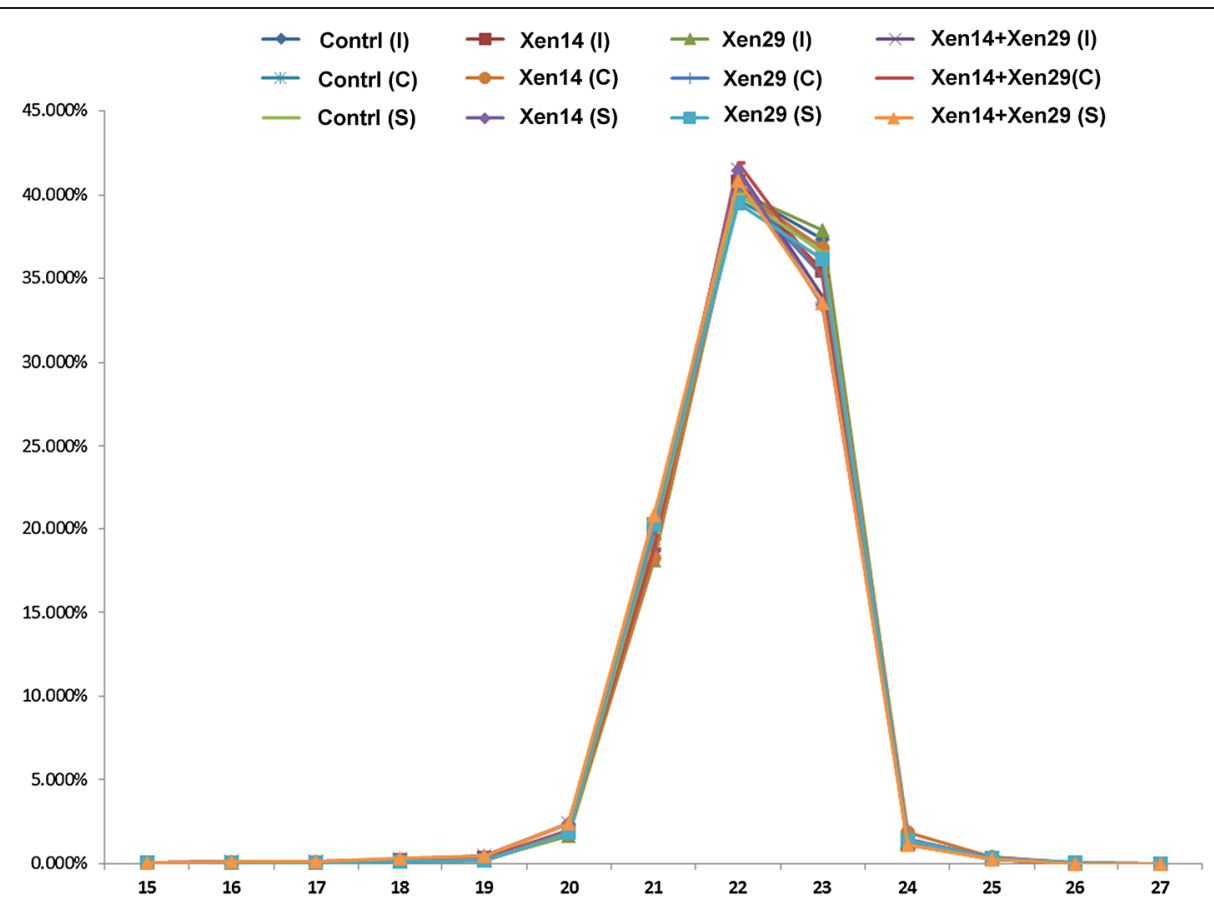

Figure 1 Length distribution and abundance of small RNA sequences by Illumina small RNA deep sequencing in 12 libraries. Xen14: recombinant-specific gram-negative pathogen Escherichia coli; Xen29: recombinant-specific gram-positive pathogen Staphylococcus aureus. Three animal models were used to create bacterial infection routes: subcutaneous injection (I), cut wound $(\mathrm{C})$, and skin graft $(\mathrm{S})$, with $1 \times 10^{8}$ Xen 14 and/or Xen29 bacteria in $100 \mu \mathrm{L}$ of phosphate buffered saline (PBS).

in the cut model, only 2 miRNAs (mir-133a-1-3p, mir133a-2-3p) were upregulated and 1 miRNA (mir-215$5 p)$ was downregulated. Exposure to mixed bacteria induced a similar expression pattern of the circulating miRNAs to that induced by gram-positive bacterial infection, but not to that induced by gram-negative bacterial infection. Unsupervised hierarchical cluster analysis (Figure 2) of all significant differentially-expressed miRNAs also revealed the mixed gram-negative and gram-positive bacteria induced a similar expression pattern of the circulating miRNAs to that induced by gram-positive bacterial infection, and gram-negative bacteria induced a similar expression pattern of the circulating miRNAs to that induced by the PBS in the control. Among these miRNAs, mir-193b-3p had the highest fold-change of 61.5-fold and 40.4-fold in the injection and skin graft models of gram-positive bacterial infection, respectively, and of 13.9-fold and 13.0-fold in the injection and skin graft models of mixed bacterial infection, followed by mir-133a-1-3p and mir-133a-2-3p in the gram-positive or mixed bacterial infection. Therefore, we focused on these 3 differentially-expressed miRNAs (mir-193b-3p, mir-133a-1-3p, mir-133a-2-3p), and their expression with sequence reads is illustrated in Figure 3. Obviously, the expression of mir-133a-1-3p and mir-133a-2-3p is similar in the 12 libraries. In addition, the high expression of mir-133a-1-3p and mir-133a-2-3p upon gram-positive bacterial infection is reduced, although still significant, in those mice with mixed bacterial infection.

\section{Discussion}

The analysis of the serum libraries from the mice receiving bacterial infection (Xen14, Xen29, and Xen14 + Xen29) against those with PBS injection by small RNA deep sequencing after $24 \mathrm{~h}$ identified differentially-expressed miRNAs. Following exposure to gram-negative bacteria alone, no differentially-expressed miRNAs were found in the injection, cut, or skin graft models. This unexpected result from gram-negative bacterial infection may be partly attributed to characteristics of the selected skin infection models (subcutaneous injection, cut, and skin graft), which were generally responsive to inoculation by the grampositive bacteria, but not the gram-negative bacteria. Investigation of the up-regulated circulating miRNAs with different kinds of animal model which facilitate a gramnegative bacteria inoculation, such as peritoneal injection, enterocolitis, or induced pneumonia may be useful to clarify the difference. In addition, the results seemed to contrast to our previous report that LPS injection induced up-regulation of the miRNAs (let-7d, miR-15b, miR-16, miR-25, miR-92a, miR-103, miR-107 and miR-451) of the 
Table 1 Differentially expressed miRNAs with sequence read $>400$ and regulated greater than 4-fold in the sera of C57BL/6 mice receiving bacterial infection for $24 \mathrm{~h}$

\begin{tabular}{|c|c|c|c|c|c|c|c|c|}
\hline \multicolumn{3}{|c|}{ Xen14: (I) Injection subcutaneously } & \multicolumn{3}{|l|}{ (C) Cut } & \multicolumn{3}{|l|}{ (G) Grafting of skin } \\
\hline miR-name & Fold-change & $p$ & miR-name & Fold-change & $p$ & miR-name & Fold-change & $p$ \\
\hline \multicolumn{3}{|l|}{ None } & \multicolumn{3}{|l|}{ None } & \multicolumn{3}{|l|}{ None } \\
\hline \multicolumn{3}{|c|}{ Xen29: (I) Injection subcutaneously } & \multicolumn{3}{|l|}{ (C) Cut } & \multicolumn{3}{|l|}{ (G) Grafting of skin } \\
\hline miR-name & Fold-change & $p$ & miR-name & Fold-change & $p$ & miR-name & Fold-change & $p$ \\
\hline mmu-mir-193b-3p & 61.5 & $* *$ & mmu-mir-133a-2-3p & 4.7 & ** & mmu-mir-193b-3p & 40.4 & ** \\
\hline mmu-mir-133a-2-3p & 21.0 & $* *$ & mmu-mir-133a-1-3p & 4.7 & ** & mmu-mir-133b-3p & 7.0 & ** \\
\hline mmu-mir-133a-1-3p & 21.0 & ** & mmu-mir-215-5p & 0.1 & $* *$ & mmu-mir-133a-2-3p & 6.6 & ** \\
\hline mmu-mir-133b-3p & 8.9 & ** & & & & mmu-mir-133a-1-3p & 6.6 & ** \\
\hline mmu-mir-434-3p & 6.2 & ** & & & & mmu-mir-434-3p & 5.7 & ** \\
\hline mmu-mir-127-3p & 5.5 & ** & & & & mmu-mir-676-3p & 5.2 & ** \\
\hline mmu-mir-676-3p & 5.0 & ** & & & & mmu-mir-127-3p & 5.1 & $* *$ \\
\hline mmu-mir-215-5p & 0.1 & ** & & & & mmu-mir-215-5p & 0.1 & ** \\
\hline \multicolumn{3}{|c|}{ Xen14 + Xen29: (I) Injection subcutaneously } & \multicolumn{3}{|l|}{ (C) Cut } & \multicolumn{3}{|l|}{ (G) Grafting of skin } \\
\hline miR-name & Fold-change & $p$ & miR-name & Fold-change & $p$ & miR-name & Fold-change & $p$ \\
\hline mmu-mir-193b-3p & 13.9 & ** & mmu-mir-133a-2-3p & 4.2 & $* *$ & mmu-mir-193b-3p & 13.0 & ** \\
\hline mmu-mir-133a-2-3p & 6.1 & ** & mmu-mir-133a-1-3p & 4.2 & $* *$ & mmu-mir-133a-1-5p & 9.6 & ** \\
\hline mmu-mir-133a-1-3p & 6.1 & $* *$ & mmu-mir-215-5p & 0.2 & $* *$ & mmu-mir-133a-2-3p & 4.7 & ** \\
\hline \multirow[t]{3}{*}{ mmu-mir-215-5p } & 0.1 & $* *$ & & & & mmu-mir-133a-1-3p & 4.7 & ** \\
\hline & & & & & & mmu-mir-133b-3p & 4.2 & ** \\
\hline & & & & & & mmu-mir-434-3p & 4.0 & ** \\
\hline
\end{tabular}

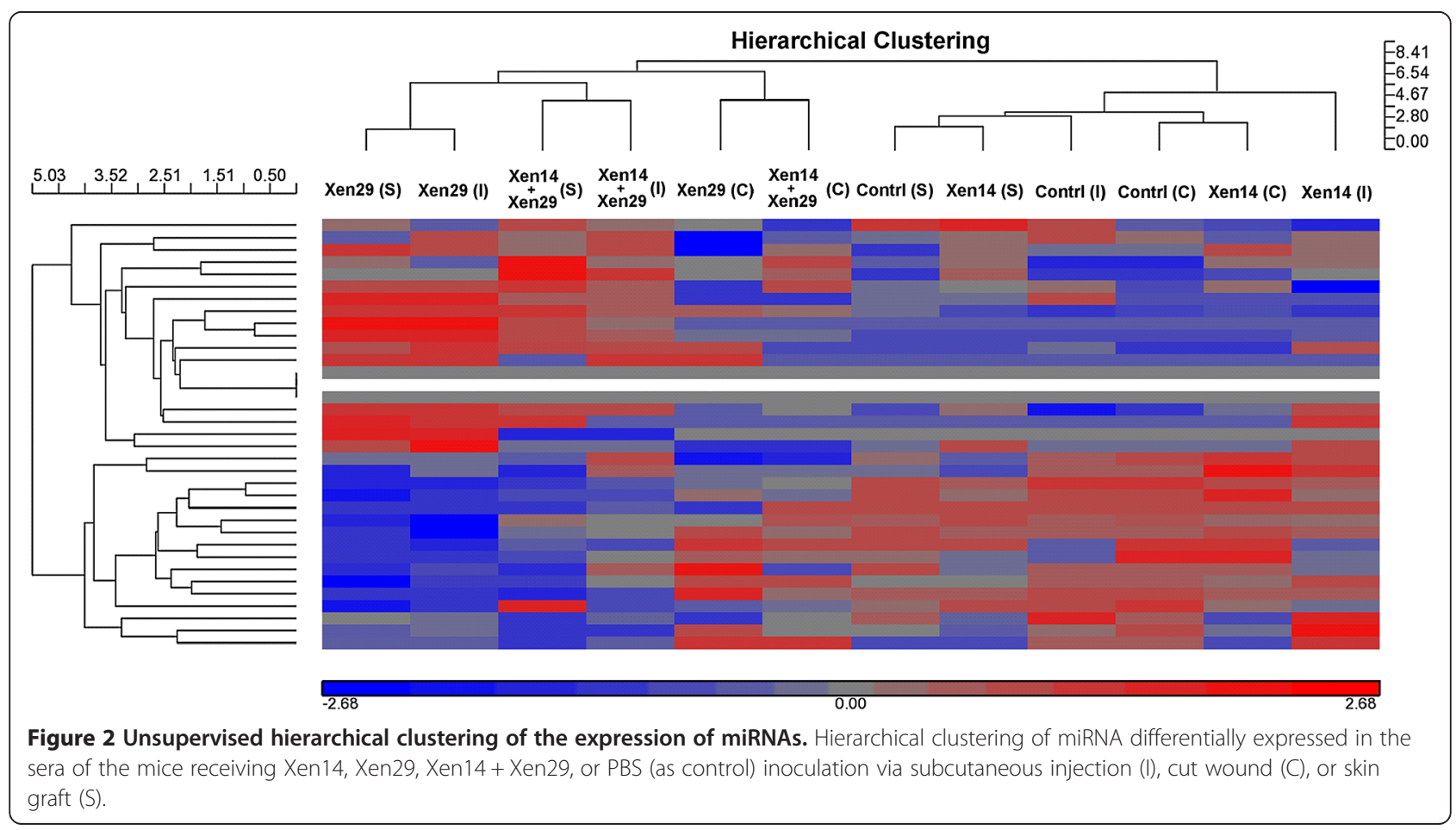




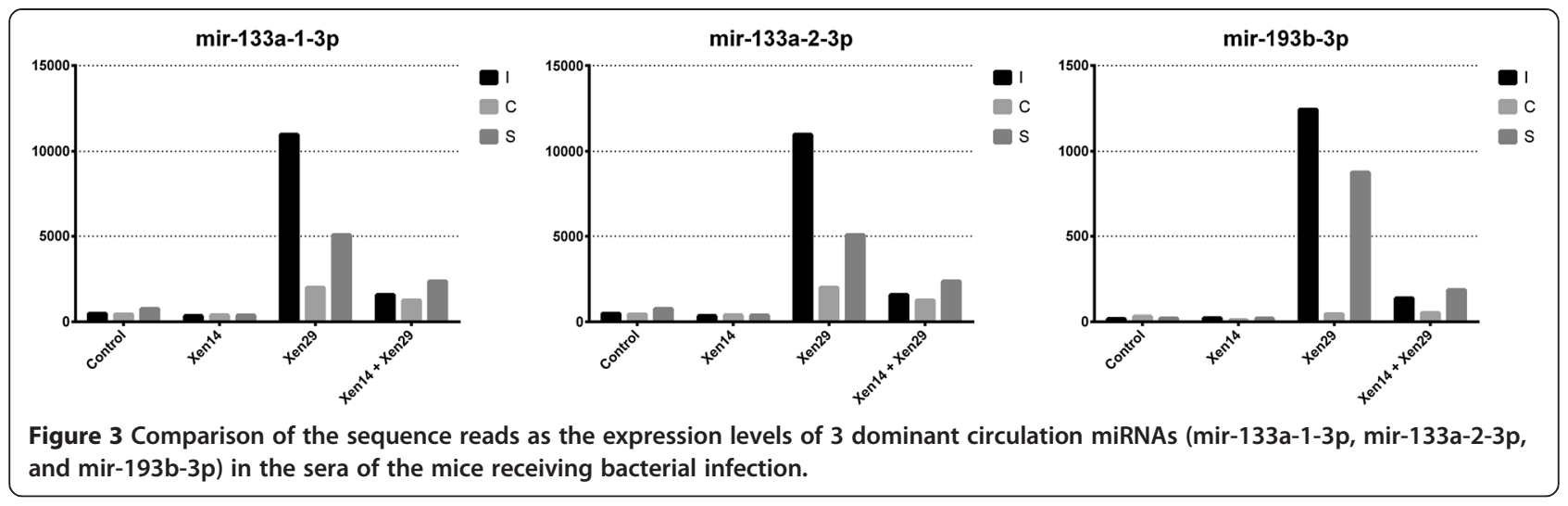

whole blood in a dose- and time-dependent manner [19]. In a single LPS injection, circulating miRNA induction occurred after $2 \mathrm{~h}$, persisted for at least $6 \mathrm{~h}$, and declined $24 \mathrm{~h}$ [19]. Considering the amount of bacteria may vary under the competition of bacterial growth and host defense after inoculation, further experiment with in vivo monitor at different observation time points may be helpful to give more information. In addition, for the same reasons, it was expected and observed in this study that the exposure to mixed bacteria induced a similar expression pattern of the circulating miRNAs to that induced by gram-positive bacterial infection. However, it is noted that the number of upregulated miRNAs and their high level of expression upon gram-positive bacterial infection was remarkably decreased in those mice with mixed bacterial infection, suggesting that the competition of different types of bacteria has an impact on the expression of circulation miRNA.

In this study, there were 8 upregulated miRNAs (mir193b-3p, mir-133a-1-3p, mir-133a-2-3p, mir-133a-1-5p, mir-133b-3p, mir-434-3p, mir-127-3p, mir-676-3p) and 1 downregulated miRNA (mir-215-5p) present as potential targets for differentiation between gram-negative and gram-positive bacterial infection. The analysis of multiple miRNAs in parallel to increase sensitivity and specificity by using complex miRNA expression patterns might constitute very useful and accessible diagnostic tools in a cluster pattern [5,7]. However, the detection of the type of infected bacteria should not be interfered from the animal model approach, particularly since the inoculation methods such as subcutaneous injection, cut, or skin grafting were not expected to have a great impact on circulating miRNA expression. Therefore, the 3 most common circulating miRNAs (mir-193b-3p, mir-133a-1-3p, and mir-133a-2-3p) expressed in the mice exposed to Staphylococcus aureus in the absence or presence of Escherichia coli may be potential biomarkers for gram-positive bacterial infection. However, whether these 3 circulating miRNAs would expressed in the mice exposed to other species of gram-positive bacteria require further validation.
A functional role of miR-133a and miR-193b was recently revealed in systemic inflammatory responses associated with infections, myocardial infarction, and cancer. Significant alterations of miR-133a, miR-193b, miR-150, and miR-155 were found in mice after cecal pole ligation and puncture-induced sepsis [12]. Although miR-193b has been considered a tumor suppressor gene, and modulates proliferation, migration, and invasion of the cancer cells [22-25], it has been reported to be associated with death from sepsis in an analysis of 214 sepsis patients (117 survivors and 97 non-survivors based on 28-day mortality) [26]. Furthermore, miR-133a is deemed a cardiac and skeletal muscle-specific miRNA $[27,28]$ and involved in muscle development [29] and many myocardial diseases [29-32]. Significantly elevated miR-133a levels were found in critically ill patients at intensive care unit (ICU) admission, especially in patients with sepsis [12]. In addition, correlation analyses revealed significant correlations of miR-133a with disease severity, classical markers of inflammation and bacterial infection, and organ failure [12]. Notably, genes encoding miR-133, namely miR-133a-1, miR-133a-2, are transcribed as bicistronic transcripts together with miR-1-1 and miR-1-2 [29]. The remarked increase in expression of mir-133a-1-3p and mir-133a-2-3p upon gram-positive bacterial infection in the present study was not accompanied by an increased expression of mir-1-1 or mir-1-2. Whether the upregulation of these circulating miRNAs is attributable to direct stimulation by gram-positive bacterial toxin or by a parallel effect, such as hemodynamic change or an associated illness, is unknown. Therefore, the origin and mechanism of the increased expression of mir-133a-1-3p and mir-133a-2-3p may require further investigation prior to their application in a clinical setting.

\section{Conclusion}

In conclusion, this study profiled circulating miRNAs in mice exposed to gram-negative and gram-positive bacteria using Illumina small RNA deep sequencing, and identified the miRNAs that may potentially be used as biomarkers of gram-positive bacterial infections. 


\section{Additional files}

Additional file 1: Table S1. Summary of Illumina small RNA deep sequencing data for small RNAs in the sera of C57BL/6 mice receiving Xen14 or/and Xen29 bacteria infection by subcutaneous injection, cut, or skin grafting. Table S2. The distribution of the sequence lengths within 15-29 nt read by the RNA deep sequencing in the 12 libraries of sera of C57BL/6 mice receiving Xen14 or/and Xen29 bacteria infection by subcutaneous injection, cut, or skin grafting. Table S3. The expression patterns of known miRNAs in the 12 libraries of sera of C57BL/6 mice receiving Xen 14 or/and Xen29 bacteria infection by subcutaneous injection, cut, or skin grafting. Table S4. The expression patterns of known miRNAs with sequence reads $>400$ in at least one of the 12 libraries of sera of C57BL/6 mice receiving Xen14 or/and Xen29 bacteria infection by subcutaneous injection, cut, or skin grafting. Table S5. The expression patterns of known miRNAs with sequence reads $>4000$ in at least one of the 12 libraries of sera of C57BL/6 mice receiving Xen 14 or/and Xen29 bacteria infection by subcutaneous injection, cut, or skin grafting. Table S6. The most top 15 expressed miRNAs with sequence reads in each of the 12 libraries of sera of $\mathrm{C} 57 \mathrm{BL} / 6$ mice receiving Xen14 or/and Xen29 bacteria infection by subcutaneous injection, cut, or skin grafting.

\section{Additional file 2: Correlation of the expression of five selected} miRNAs in small RNA deep sequencing and qPCR.

\section{Competing interests}

The authors have no personal financial or institutional interest in any of the drugs, materials, or devices described in this article.

\section{Authors' contributions}

$\mathrm{CHH}$ and CSR were responsible for the design and coordination of the data acquisition and analysis, and the writing of the manuscript. SCW and JCY participated by providing and coordinating the resources. YCC and CJW were involved in the acquisition of the deep sequencing data. THL, YCW, and SLT contributed to the animal surgery and acquisition of the study specimens. All authors read and approved the final manuscript.

\section{Acknowledgements}

The work was supported by Chang Gung Memorial Hospital (CMRPG8C1101) and by National Science Council (NMRPG8D6111), Taiwan. We thank the analysis work supported by Genomic \& Proteomic Core Laboratory, Department of Medical Research, Kaohsiung Chang Gung Memorial Hospital.

\section{Author details}

'Department of Neurosurgery, Kaohsiung Chang Gung Memorial Hospital and Chang Gung University College of Medicine, Kaohsiung City 833, Taiwan. ${ }^{2}$ Department of Anesthesiology, Kaohsiung Chang Gung Memorial Hospital and Chang Gung University College of Medicine, Kaohsiung City 833, Taiwan. ${ }^{3}$ Department of Plastic and Reconstructive Surgery, Kaohsiung Chang Gung Memorial Hospital and Chang Gung University College of Medicine, No.123, Ta-Pei Road, Niao-Song District, Kaohsiung City 833, Taiwan.

Received: 14 August 2014 Accepted: 15 December 2014 Published online: 07 January 2015

\section{References}

1. Stefani G, Slack FJ. Small non-coding RNAs in animal development. Nat Rev Mol Cell Biol. 2008;9(3):219-30.

2. Macha MA, Seshacharyulu P, Krishn SR, Pai P, Rachagani S, Jain M, et al. MicroRNAs (miRNA) as Biomarker(s) for prognosis and diagnosis of gastrointestinal (GI) cancers. Curr Pharm Design. 2014;20(33):5287-97.

3. Rao P, Benito E, Fischer A. MicroRNAs as biomarkers for CNS disease. Front Mol Neurosci. 2013;6:39.

4. Tousoulis D, Androulakis E, Papageorgiou N, Siasos G, Latsios G, Charakida $M$, et al. Novel biomarkers assessing endothelial dysfunction: role of microRNAs. Curr Top Med Chem. 2013;13(13):1518-26.

5. Gilad S, Meiri E, Yogev Y, Benjamin S, Lebanony D, Yerushalmi N, et al. Serum microRNAs are promising novel biomarkers. PLoS One. 2008;3(9): e3148.
6. Kosaka N, Iguchi H, Ochiya T. Circulating microRNA in body fluid: a new potential biomarker for cancer diagnosis and prognosis. Cancer Sci. 2010;101(10):2087-92.

7. Chen X, Ba Y, Ma L, Cai X, Yin Y, Wang K, et al. Characterization of microRNAs in serum: a novel class of biomarkers for diagnosis of cancer and other diseases. Cell Res. 2008;18(10):997-1006.

8. Mitchell PS, Parkin RK, Kroh EM, Fritz BR, Wyman SK, Pogosova-Agadjanyan EL, et al. Circulating microRNAs as stable blood-based markers for cancer detection. Proc Natl Acad Sci U S A. 2008;105(30):10513-8.

9. Zeng L, Cui J, Wu H, Lu Q. The emerging role of circulating microRNAs as biomarkers in autoimmune diseases. Autoimmunity. 2014;47(7):419-29.

10. Sayed AS, Xia K, Salma U, Yang T, Peng J. Diagnosis, prognosis and therapeutic role of circulating miRNAs in cardiovascular diseases. Heart Lung Circ. 2014;23(6):503-10.

11. Wang HJ, Zhang PJ, Chen WJ, Jie D, Dan F, Jia YH, et al. Characterization and Identification of novel serum microRNAs in sepsis patients with different outcomes. Shock (Augusta, Ga). 2013;39(6):480-7.

12. Tacke F, Roderburg C, Benz F, Cardenas DV, Luedde M, Hippe HJ, et al. Levels of circulating miR-133a are elevated in sepsis and predict mortality in critically ill patients. Crit Care Med. 2014;42(5):1096-104.

13. Qi P, Cheng SQ, Wang H, Li N, Chen YF, Gao CF. Serum microRNAs as biomarkers for hepatocellular carcinoma in Chinese patients with chronic hepatitis B virus infection. PLoS One. 2011;6(12):e28486.

14. Zhang X, Zhang Z, Dai F, Shi B, Chen L, Zhang X, et al. Comparison of circulating, hepatocyte specific messenger RNA and microRNA as biomarkers for chronic hepatitis B and C. PLoS One. 2014;9(3):e92112.

15. Miotto P, Mwangoka G, Valente IC, Norbis L, Sotgiu G, Bosu R, et al. miRNA signatures in Sera of patients with active pulmonary tuberculosis. PLoS One. 2013;8(11):e80149.

16. Manzano-Roman R, Siles-Lucas M. MicroRNAs in parasitic diseases: potentia for diagnosis and targeting. Mol Biochem Parasitol. 2012;186(2):81-6.

17. Tritten L, Burkman E, Moorhead A, Satti M, Geary J, Mackenzie C, et al. Detection of circulating parasite-derived MicroRNAs in Filarial infections. PLoS Negl Trop Dis. 2014;8(7):e2971.

18. Hsieh CH, Yang JC, Jeng JC, Chen YC, Lu TH, Tzeng SL, et al. Circulating microRNA signatures in mice exposed to lipoteichoic acid. J Biomed Sci. 2013;20:2.

19. Hsieh CH, Rau CS, Jeng JC, Chen YC, Lu TH, Wu CJ, et al. Whole blood-derived microRNA signatures in mice exposed to lipopolysaccharides. J Biomed Sci. 2012;19:69.

20. Fabbri M. TLRs as miRNA receptors. Cancer Res. 2012;72(24):6333-7.

21. Van Nieuwerburgh F, Soetaert S, Podshivalova K, Ay-Lin Wang E, Schaffer L, Deforce D, et al. Quantitative bias in illumina TruSeq and a novel post amplification barcoding strategy for multiplexed DNA and small RNA deep sequencing. PLOS One. 2011;6(10):e26969.

22. Xie C, Jiang XH, Zhang JT, Sun TT, Dong JD, Sanders AJ, et al. CFTR suppresses tumor progression through miR-193b targeting urokinase plasminogen activator (UPA) in prostate cancer. Oncogene. 2013;32(18):2282-91. 2291.e2281-2287.

23. Hu H, Li S, Liu J, Ni B. MicroRNA-193b modulates proliferation, migration, and invasion of non-small cell lung cancer cells. Acta Biochim Biophys Sin. 2012;44(5):424-30.

24. Chen J, Zhang X, Lentz C, Abi-Daoud M, Pare GC, Yang X, et al. miR-193b regulates Mcl-1 in melanoma. Am J Pathol. 2011;179(5):2162-8.

25. Xu C, Liu S, Fu H, Li S, Tie Y, Zhu J, et al. MicroRNA-193b regulates proliferation, migration and invasion in human hepatocellular carcinoma cells. Eur J Cancer. 2010;46(15):2828-36.

26. Wang $H$, Zhang $P$, Chen W, Feng D, Jia Y, Xie L. Serum microRNA signatures identified by Solexa sequencing predict sepsis patients' mortality: a prospective observational study. PLoS One. 2012;7(6):e38885.

27. Jeng SF, Rau CS, Liliang PC, Wu CJ, Lu TH, Chen YC, et al. Profiling musclespecific microRNA expression after peripheral denervation and reinnervation in a rat model. J Neurotrauma. 2009;26(12):2345-53.

28. Townley-Tilson WH, Callis TE, Wang D. MicroRNAs 1, 133, and 206: critical factors of skeletal and cardiac muscle development, function, and disease. Int J Biochem Cell Biol. 2010;42(8):1252-5.

29. Yu H, Lu Y, Li Z, Wang Q. MicroRNA-133: Expression, function and therapeutic potential in muscle diseases and cancer. Curr Drug Targets. 2014;15(9):817-28

30. Ning B, Qi X, Li Y, Liu H, Zhang F, Qin C. Biventricular pacing cardiac contractility modulation improves cardiac contractile function via 
upregulating SERCA2 and miR-133 in a rabbit model of congestive heart failure. Cell Physiol Biochem. 2014;33(5):1389-99.

31. Peng L, Chun-guang Q, Bei-fang L, Xue-zhi D, Zi-hao W, Yun-fu L, et al. Clinical impact of circulating miR-133, miR-1291 and miR-663b in plasma of patients with acute myocardial infarction. Diagn Pathol. 2014;9:89.

32. Care A, Catalucci D, Felicetti F, Bonci D, Addario A, Gallo P, et al. MicroRNA-133 controls cardiac hypertrophy. Nat Med. 2007;13(5):613-8.

Submit your next manuscript to BioMed Central and take full advantage of:

- Convenient online submission

- Thorough peer review

- No space constraints or color figure charges

- Immediate publication on acceptance

- Inclusion in PubMed, CAS, Scopus and Google Scholar

- Research which is freely available for redistribution 